Manuskript „Harnableitung beim älteren Patienten” für die Zeitschrift Der Urologe 12/2015

\title{
DOI 10.1007/s00120-015-4010-1
}

Korrespondenzadresse: PD Dr. M. Spahn

Urologische Universitätsklinik, Inselspital Bern

Anna Seiler-Haus, CH-3010 Bern, Schweiz

martin.spahn@insel.ch

Weitere Autoren: $\quad$ S. Boxler

$\checkmark \quad$ Bitte kreuzen Sie die gewünschten Optionen an und senden Sie dieses Formular an die oben angegebene E-Mail-Adresse oder Faxnummer. Dies ist auch dann wichtig, wenn Sie keine Korrekturen haben. Vielen Dank. Die Erteilung der Rechtseinräumung mit Ihrer Unterschrift ist für eine Veröffentlichung erforderlich!

\section{Imprimatur}

Bitte beachten Sie, dass nach Imprimatur keine weiteren Textkorrekturen, Änderungen der Autorenschaft oder der

Autorenreihenfolge möglich sind.

Folgende Korrekturmöglichkeiten stehen Ihnen offen:

- Fügen Sie Ihre Korrekturen komfortabel mit der Kommentarfunktion Ihres PDF-Programms (z.B. Adobe Professional)

direkt ins Beitrags-PDF ein.

- Alternativ können Sie das PDF ausdrucken und Ihre Korrekturen gut lesbar in den Ausdruck einfügen.

Hiermit gebe ich mein Manuskript direkt frei. Korrekturen sind nicht notwendig.

Ich habe einzelne Seiten des Manuskriptes korrigiert und diese der Antwort beigefügt. Nach ausgeführter Korrektur ist das Manuskript imprimiert.

Folgende Manuskript-Seiten habe ich beigefügt:

$\square \quad$ Diese Rückantwort enthält das komplette Manuskript mit entsprechenden Korrekturwünschen. Nach ausgeführter Korrektur ist das Manuskript imprimiert. Mir ist bewusst, dass größere Korrekturen oder Änderungen an der Textlänge ein späteres Publikationsdatum nach sich ziehen können.

\section{Sonderdrucke}

Ich wünsche kostenpflichtige Sonderdrucke meines veröffentlichten Manuskriptes. Das ausgefüllte Formular "Sonderdruckbestellung“ liegt bei.

\section{OpenAccess}

Springer bietet Ihnen die Möglichkeit, Ihren Artikel Open Access über das Springer Open Choice Programm zu veröffentlichen. Ihr Beitrag wird dann sofort und dauerhaft für jedermann weltweit online freiverfügbar. Dies ist der einfachste Weg für Sie Open Access Mandaten zu entsprechen, da der Beitrag unter der liberalen Creative Commons Attribution (CC BY) Lizenz publiziert wird, welche die freie Distribution und Weiternutzung der finalen Version unterstützt. Die einmalige Open Access Gebühr beträgt EUR 2.200 (netto).

Ich wünsche nähere Informationen über die Möglichkeit, mein Manuskript Open Access zu publizieren.

\section{Einhaltung ethischer Richtlinien}

\section{IVa Interessenkonflikt}

Die Angabe eines Interessenkonflikts ist seriöse Publikationspraxis. Bitte geben Sie alle finanziellen oder persönlichen Beziehungen von Ihnen und Ihren Koautoren zu Dritten an, deren Interessen vom Beitragsinhalt positiv oder negativ betroffen sein können, auch wenn aus Ihrer Sicht keine Beeinflussung stattgefunden hat. Beispiele: finanzielle Verbindung zu Pharmafirmen, Beschäftigungsverhältnisse, Beratungs- und Referententätigkeiten, Honorare, Reisekostenübernahmen, Drittmittel, Aktienbesitz. Die Erklärung bezieht sich auf die Gegenwart und die vergangenen 5 Jahre und wird veröffentlicht.

Auch wenn kein Interessenkonflikt besteht, ist dies anzugeben. Sollte der Interessenkonflikt bereits im Manuskript genannt worden sein, erübrigt sich die Angabe an dieser Stelle.

Der korrespondierende Autor gibt für sich und seine Koautoren an, dass kein Interessenkonflikt besteht.

Der korrespondierende Autor weist für sich und seine Koautoren auf folgende Beziehung/en hin (Bitte machen Sie separate Angaben zu jedem Autor auch wenn nicht für jeden Autor ein Interessenkonflikt besteht): 
IVb Patientenrechte und Tierschutzbestimmungen

Medizinische Forschung unterliegt ethischen Standards, die die Achtung vor den Menschen fördern und ihre Gesundheit und Rechte schützen. Ärzte sollen die ethischen, rechtlichen und behördlichen Normen und Standards für Forschung am Menschen und am Tier ihrer eigenen Länder sowie die maßgeblichen internationalen Normen und Standards berücksichtigen.

Bitte machen Sie im Folgenden die für das vorliegende Manuskript zutreffenden Angaben.

$\square \quad$ Hiermit bestätige ich als korrespondierender Autor, dass das vorliegende Manuskript keine Studien an Menschen oder Tieren enthält.

Falls Patienten durch Abbildungen oder anderweitige Angaben im Manuskript zu identifizieren sind:

$\square \quad$ Alle Patienten, die über Bildmaterial oder anderweitige Angaben innerhalb des Manuskripts zu identifizieren sind, haben hierzu ihre schriftliche Einwilligung gegeben. Im Falle von nicht mündigen Patienten liegt die Einwilligung eines Erziehungsberechtigten oder des gesetzlich bestellten Betreuers vor.

Falls dem vorliegenden Manuskript Studien am Menschen zugrunde liegen:

$\square \quad$ Alle im vorliegenden Manuskript beschriebenen Untersuchungen am Menschen wurden mit Zustimmung der zuständigen Ethik-Kommission, im Einklang mit nationalem Recht sowie gemäß der Deklaration von Helsinki von 1975 (in der aktuellen, überarbeiteten Fassung) durchgeführt. Von allen beteiligten Patienten liegt eine Einverständniserklärung vor.

Falls dem vorliegenden Manuskript Experimente mit Labortieren zugrunde liegen:

$\square \quad$ Hiermit bestätige ich als korrespondierender Autor, dass alle nationalen Richtlinien zur Haltung und zum Umgang mit Labortieren eingehalten wurden und die notwendigen Zustimmungen der zuständigen Behörden vorliegen.

\section{Rechtseinräumung}

In Erweiterung des §38 Abs. 1 UrhG räume ich als erstgenannter Verfasser der Springer Science+Business Media Gruppe das ausschließliche, geografisch und zeitlich unbeschränkte Recht der Speicherung, Vervielfältigung, Verbreitung und Wiedergabe meines Beitrages (inkl. Bilder, Video- und Audiodateien) ein. Dies schließt das Recht zur Übersetzung und Bearbeitung ein und gilt für die Dauer des gesetzlichen Urheberrechts für alle Auflagen und Publikationen in gedruckter und elektronischer Form (z.B. online, offline, mobile Nutzung, embedded content). Springer ist berechtigt, die Nutzungsrechte am gesamten Beitrag und Teilen daraus in gedruckter und elektronischer Form wahrzunehmen und weiterzugeben.

Hiermit versichere ich - auch im Namen der Miturheber -, dass ich berechtigt bin, über die urheberrechtlichen Nutzungsrechte an dem o.g. Beitrag zu verfügen. 


\section{Autorenfortdrucke - Bestellung}

Wenn Sie Fortdrucke Ihres Artikels bestellen möchten, füllen Sie bitte dieses Formular aus und senden es zusammen mit Ihren Korrekturen an uns zurück. Bitte beachten Sie, dass alle Anzeigen, die in der gedruckten Version Ihres Beitrags im Heft veröffentlicht werden, auch im Sonderdruck erscheinen. Die Lieferzeit für Fortdrucke beträgt produktionsbedingt 3 bis 6 Wochen.

Sollten Sie erst nach dem Druck eines Heftes Fortdrucke bestellen, beträgt die Mindestabnahme 100 Exemplare, da hier ein erhöhter Aufwand zu berücksichtigen ist. Diese Sonderdrucke werden als kommerzielle Sonderdrucke kalkuliert.

Achtung: Ihre Bestellung kann nur bearbeitet werden, wenn eine Kreditkartennummer und Unterschrift vorliegen.

Hiermit bestelle ich:

\begin{tabular}{|l|l|}
\hline$\square \mathbf{5 0}$ Exemplare & 300 EUR \\
\hline$\square \mathbf{1 0 0}$ Exemplare & 365 EUR \\
\hline$\square \mathbf{2 0 0}$ Exemplare & 525 EUR \\
\hline$\square \mathbf{3 0 0}$ Exemplare & 680 EUR \\
\hline (Preise inkl. Porto und Verpackung, zzgl. MwSt.)
\end{tabular}

Für die Bestellung von mehr als 300 Exemplaren, oder von Sonderdrucken, die nach Druck des Heftes produziert werden, wenden Sie sich bitte an:

Kontakt Reprints

Christoph Holzhaus

Telefon 030 / 827 87-57 14, Fax -6 5714

E-Mail christoph.holzhaus@springer.com

Bitte geben Sie Ihre VAT-Nummer an:

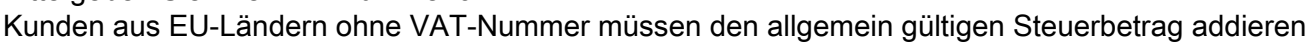

Bitte geben Sie Ihre Kreditkartennummer an:

$\square$ Eurocard/Access/Mastercard

$\square \quad$ American Express

$\square \quad$ Visa/Barclaycard/BankAmericard

Kartennummer (inkl. Prüfziffern):

Gültig bis: ${ }_{-} / \ldots$

Datum / Unterschrift:
Für Autoren wohnhaft in Deutschland ist die Zahlung per Bankeinzug möglich:

Hiermit erteile ich Springer die

Abbuchungsgenehmigung von meinem Bankkonto über den Rechnungsbetrag bei Fälligkeit.

Kontonr.:

Bankleitzahl:

Bank:

Datum / Unterschrift:

\section{Rechnungsanschrift:}

$\square \quad$ siehe Lieferanschrift

$\square$

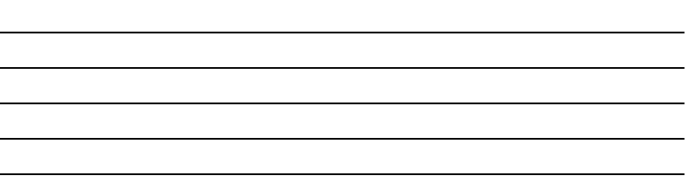

\section{Lieferung an:}

$\square \quad$ PD Dr. M. Spahn Urologische Universitätsklinik Inselspital Bern Anna Seiler-Haus $\mathrm{CH}-3010$ Bern Schweiz

Bitte verwenden Sie bei der Rechnungsstellung folgende Bestellnummer/folgendes Zeichen:

Bitte überprüfen Sie die oben angegebenen Daten und korrigieren Sie sie gegebenfalls. 
- IV - 


\begin{tabular}{|l|l|}
\hline DOI & $10.1007 /$ s00120-015-4010-1 \\
\hline Copyright & Springer-Verlag Berlin Heidelberg - 2015 \\
\hline
\end{tabular}

\title{
Leitthema
}

\section{Harnableitung beim älteren Patienten}

\author{
M. Spahn · S. Boxler \\ Urologische Universitätsklinik, Inselspital Bern, Bern, Schweiz
}

\section{Korrespondenzadresse}

PD Dr. M. Spahn

Urologische Universitätsklinik, Inselspital Bern

Anna Seiler-Haus

$\mathrm{CH}-3010$ Bern

Schweiz

martin.spahn@insel.ch

Online publiziert: Onlinedatum erscheint nach Freigabe

\section{Zusammenfassung}

Hintergrund Das Blasenkarzinom ist eine der 10 häufigsten Krebserkrankungen mit weltweit über 400.000 Neuerkrankungen/Jahr. Patienten mit muskelinvasiven Blasentumoren sterben ohne Behandlung in $80 \%$ der Fälle innerhalb von 2 Jahren.

Methode Die Zystektomie stellt die Therapie der Wahl dar. Das mediane Alter der neu diagnostizierten Patienten liegt bei 70 Jahren und somit sind eher ältere Patienten davon betroffen. Demographisch bedingt wird die Anzahl Zystektomien von älteren Patienten in den nächsten Jahren zunehmen. Die Wahl der Harnableitung stellt bezüglich perioperativer Morbidität und nachfolgender Lebensqualität einen entscheidenden Faktor dar. Am häufigsten werden bei älteren Patienten inkontinente Harnableitungen angelegt.

Schlussfolgerung Das Alter allein keine Kontraindikation für die orthotope ileale Ersatzblase oder einen katheterisierbaren Pouch dar. Der Patientenselektion und einer nervenschonenden 
Operationstechnik kommt beim älteren Patienten beiderlei Geschlechts eine entscheidende Bedeutung zu, um exzellenten funktionelle Ergebnissen zu erzielen.

\section{Schlüsselwörter}

Blasenkarzinom $\cdot$ Ersatzblase, orthotope $\cdot$ Zystektomie $\cdot$ Ileozäkalpouch $\cdot$ Pouch, katheterisierbarer

\section{Urinary diversion in elderly patients}

\section{Abstract}

Background Bladder cancer represents one of the ten most prevalent cancers worldwide. More than 400,000 people worldwide are newly diagnosed every year. Within 2 years after diagnosis, $80 \%$ of patients with muscle invasive bladder cancer without treatment die.

Methods The aggressive local surgical approach with a cystectomy is the therapy of choice. The median age of patients with de novo bladder cancer is 70 years. Thus bladder cancer is a cancer of the elderly. For demographical reasons, the number of eldery patients undergoing radical cystectomy will rise in the next few years. The type of urinary diversion is a major factor influencing perioperative morbidity and quality of life in these patients. Incontinent urinary diversions are preferentially used in daily practice.

Conclusions There are only a few contraindications for orthotopic neobladder; however, age alone is not a contraindication. Patient selection and a nerve sparing approach are crucial in men and women to achieve excellent functional results with orthotopic neobladder in elderly patients.

\section{Keywords}

Urinary bladder neoplasms $\cdot$ Neobladder, orthotopic $\cdot$ Cystectomy $\cdot$ Ileocecal pouch $\cdot$ Pouch, catheterizable

Das Blasenkarzinom ist eine der 10 häufigsten Krebserkrankungen mit weltweit über 400.000 Neuerkrankungen/Jahr. Patienten mit muskelinvasiven Blasentumoren sterben ohne Behandlung in $\mathbf{8 0 \%}$ der Fälle innerhalb von 2 Jahren. Die Zystektomie stellt die Therapie der 
Wahl dar. Aufgrund des altersabhängigen Auftretens und der demographischen Entwicklung wird die Anzahl Zystektomien bei älteren Patienten in den nächsten Jahren deutlich zunehmen. Der Form der Harnableitung beim älteren Patienten kommt eine entscheidende Bedeutung im Erhalt der Lebensqualität zu.

Das Urothelkarzinom der Harnblase ist die neunthäufigste Krebserkrankung, mit 429.800 Neuerkrankungen weltweit im Jahr 2012 [1]. Die altersadaptierte Inzidenzrate des Harnblasenkarzinoms in Westeuropa beträgt für die männliche Bevölkerung 19,7/100.000 Einwohner und 4,3 für die weibliche Bevölkerung [1]. Etwa 20-30\% der Harnblasenkrebspatienten weisen bei Diagnosestellung einen muskelinvasiven Tumor mit oder ohne Metastasennachweis auf. Ohne aggressive lokale oder systemische Behandlung versterben $>80 \%$ dieser Patienten innerhalb von 2 Jahren an ihrem Tumor und mehr als die Hälfte der Patienten entwickelt in Abhängigkeit vom Tumorstadium trotz aggressiver Behandlungen innerhalb von 5 Jahren ein Rezidiv mit Fernmetastasen $[\underline{2}, \underline{3}, \underline{4}]$.

\section{$>$ Eine Lebenserwartung von 2 Jahren ist als Indikation für die Zystektomie akzeptiert}

Die bei anderen Tumorerkrankungen häufig verwendete 10-Jahres-Lebenserwartung zur Planung weiterer Behandlungen ist beim Harnblasenkarzinom somit nicht anwendbar. Eine allgemeine Lebenserwartung von 2 Jahren ist als Indikation für die radikale Zystektomie akzeptiert. Auch wenn das Blasenkarzinom in jeder Altersgruppe auftreten kann, so handelt es sich in der Mehrzahl der Fälle um eine Erkrankung des älteren Menschen. Das mediane Patientenalter bei Diagnosestellung liegt bei 70 Jahren.

Aufgrund der zunehmenden Lebenserwartung und dem rasch ansteigenden Anteil der älteren Bevölkerung ist für die Zukunft mit einer überproportionalen Zunahme älterer und sehr alter Patienten mit Harnblasenkarzinom zu rechnen die einer kurativen oder palliativen Therapie bedürfen. Dem operativen Management des Harnblasenkarzinoms beim älteren Menschen kommt somit in der Zukunft eine zunehmende Bedeutung zu. Die Wahl der Harnableitung stellt hierbei einen wichtigen Aspekt dar, um eine hohe Lebensqualität zu erreichen.

\section{Der ältere Patient - wer ist das?}

Häufig werden Patienten ab einem Alter von 65 Lebensjahren als ,älter“ bezeichnet. Die Grundlage, auf welcher diese Definition basiert, ist weitgehend unklar, sie liegt aber 
vermutlich zumindest teilweise in der von Prinz Bismack vor mehr als einem Jahrhundert festgelegten Altersgrenze von 65 Jahren, die zum Empfang von Rentenzahlungen aus dem nationalen Pensionsplan berechtigten. Diese Definition hat sich geändert und ändert sich noch immer. Nimmt man das chronologische Alter, so zeigt sich über die Zeit eine Verschiebung der Definition was „alt“ ist in Richtung einer höheren Anzahl von Lebensjahren. Die Medizin trägt diesen Veränderungen durch eine Unterscheidung zwischen ,young old“ (65-74 Jahre), „old old“ (75-84 Jahre) und „oldest old“ ( $\geq 85$ Jahre) Rechnung. Neben dem chronologischen Alter spielt aber das physiologische Alter eine wesentliche Rolle. Mit zunehmendem chronologischem Alter nehmen unabhängig von parallel vorhandenen Erkrankungen die funktionellen Reserven praktisch aller Organsysteme ab. Verschiedene Erkrankungen addieren sich auf diese altersphysiologischen normalen Veränderungen, die dann für den betroffenen belastend werden, wenn sie ihn in seiner Funktionalität einschränken oder z. B. im Rahmen von Operationen an die Grenzen der Belastbarkeit bringen [5] .

Das Hauptziel bei der medizinischen und chirurgischen Behandlung älterer Patienten ist der Erhalt der Funktionalität, der größtmöglichen Selbstständigkeit und damit verbunden der Lebensqualität. Dazu bedarf es in der Planung einer Zystektomie einer differenzierten individuellen Risikoabschätzung und einer Priorisierung bei Multimorbidität, welche in einem interdisziplinären Team erfolgen sollte.

\section{Verhältnis zwischen Alter, Komplikationsraten und Mortalität der radikalen Zystektomie mit Harnableitung}

Die radikale chirurgische Entfernung der Harnblase ist die Standardtherapie des muskelinvasiven Blasenkarzinoms. Verbesserungen in der Operationstechnik und dem anästhesiologischen sowie perioperativen Management haben in den vergangenen Dekaden zu einem deutlichen Rückgang der Mortalitätsrate von 20\% auf etwa 2\% geführt [므, $\underline{6}]$.

Dennoch weist die radikale Zystektomie auch heute noch ein signifikantes Morbiditätsrisiko mit einer Gesamtkomplikationsrate von 10-50\% auf. Eine Reihe Publikationen konnte eine signifikante Korrelation zwischen Alter und der Häufigkeit perioperativer Komplikationen nachweisen [7]. Die 90-Tage-Mortalitätsraten älterer Patienten nach Zystektomie variieren zwischen 5 und 15\% [고 8, $\underline{9}]$. Welchen Einfluss die jeweilig gewählte Harnableitung auf die Häufigkeit perioperativer Komplikationen bei älteren Patienten hat, wurde nur selten 
getrennt analysiert. In gut selektierten Patienten zeigte sich jedoch in Exzellenzzentren kein Unterschied bezüglich der Häufigkeit und der Schwere perioperativer Komplikationen bei älteren Patienten $[\underline{10}, \underline{11}]$.

\section{Welche Harnableitung bei welchem Patienten?}

Das Ziel der operativen Behandlung von Patienten mit muskelinvasivem Harnblasenkarzinom ist ein gutes onkologisches Ergebnis unter Erhalt der Lebensqualität. Exzellente funktionelle Ergebnisse sind hierbei ebenso wichtig wie die Langzeitremission. Der gewählten Harnableitung kommt hierbei besonders im fortgeschrittenen Alter eine entscheidende Bedeutung zu. Die individuelle Tumorkonstellation, Begleiterkrankungen, die psychische und körperliche Situation, das soziale Umfeld, die Compliance, aber auch der Patientenwunsch sollten bei der Wahl der Harnableitung Berücksichtigung finden.

\section{$>$ Der gewählten Harnableitung kommt im fortgeschrittenen Alter eine entscheidende Bedeutung zu}

Das Patientenalter als alleiniges Kriterium sollte die Möglichkeit einer kontinenten Harnableitung nicht ausschließen. Dennoch zeigt die tägliche Praxis, dass die Mehrzahl der älteren Patienten (70\%) eine inkontinente Harnableitung, mehrheitlich in Form eines Ileumconduits, erhält. Lediglich 30\% der Patienten werden mit einer kontinenten Harnableitung, meist in Form einer orthotopen ilealen Erstazblase, versorgt [7]. Allgemeine Richtlinien, welcher Patient mit welcher Harnableitung am besten behandelt wird, gibt es nicht. Dem betreuenden Team kommt daher in der Risikoeinschätzung und der individuellen Beratung des Patienten eine entscheidende Rolle zu. Sicher profitieren Patienten mit schweren Begleiterkrankungen oder die Patienten mit nicht kurierbarem Tumor, die zur Symptomkontrolle eine Zystektomie erhalten müssen, nicht von einer kontinenten Harnableitung. In diesen Fällen ist die Anlage einer Ureterokutaneostomie eine vertretbare Lösung. Diese gewährleistet eine kurze Operationszeit mit geringst möglichem Operationstrauma und damit einhergehend geringen Komplikationsraten [7]].

Die Indikation und die Selektionskriterien für eine orthotope ileale Ersatzblase wurde in den vergangenen Dekaden immer mehr ausgeweitet. Heutzutage bestehen nur noch wenige Kontraindikationen (Tab. 1). Zu diesen zählt der Tumorbefall der distalen prostatischen Harnröhre mit erhöhtem Lokalrezidivrisikos, eine eingeschränkte Nierenfunktion 
[Serumkreatinin $>150 \mu \mathrm{mol} / 1$ oder glomeruläre Filtrationsrate (GFR) $<50 \mathrm{ml} / \mathrm{min}$ ], sofern diese nicht durch eine tumorbedingte Ureterobstruktion verursacht ist, die eine gute Chance hat sich nach Anlage einer perkutanen Nephrostomie zu normalisieren. Schwere Leberfunktionsstörungen bergen ein signifikantes Risiko für das Auftreten einer hyperammoniämischen Enzephalopathie und stellen neben entzündlichen Darmerkrankungen, Sphinkterdysfunktionen, Harninkontinenz, rezidivierenden Harnröhrenstrikturen und stattgehabten Bestrahlungen im kleinen Beckens, die mit einer erhöhten Inkontinenzgefahr einhergehen, weitere Kontraindikationen dar. In diesen Fällen ist die Anlage eines Ileumconduits eine geeignete Lösung für ältere Patienten.

Bestehen diese Kontraindikationen nicht, so ist der Patient prinzipiell ein Kandidat für eine kontinente Harnableitung. Das Alter allein stellt keine Kontraindikation dar. Relativ robuste Zahlen zeigen bei Ersatzblasenpatienten über alle Altersgruppen hinweg Kontinenzraten zwischen 85 und 90\% (trocken/1 Pad/Tag, 12 Monate postoperativ), [12]. Obgleich eine inverse Korrelation zwischen Patientenalter und Kontinenz nach orthotopem Blasenersatz nachweisbar ist, liegen nur wenige Daten über die funktionellen Ergebnisse bei älteren Patienten vor. Saika et al. [13] berichteten über Kontinenzraten von 50\% bei über 75-jährigen Männern mit orthotopem Blasenersatz. Sogni et al. [11] berichteten über 56\% Kontinenz am Tage und 25\% nächtliche Kontinenz. Interessanterweise führten $70 \%$ der Patienten in dieser Serie einen sterilen intermittierenden Einmalkatheterismus durch, was in dieser Altersgruppe zu Problemen führen kann. Dennoch berichten Zentren mit großer Erfahrung über deutlich bessere Ergebnisse in selektierten älteren Patienten $(>90 \%$ Tageskontinenz und $80 \%$ nächtliche Kontinenz bei über 65-Jährigen), [14]. Wüthrich et al. [9] bestätigten diese Ergebnisse kürzlich an einem sorgfältig selektierten Patientengut von 35 über 75-jährigen Patienten, die eine 86\%ige Kontinenz am Tage (trocken/1 Pad/Tag) und eine 72\%ige nächtliche Kontinenz zeigten. Der richtigen Patientenselektion kommt demnach eine entscheidende Bedeutung zu. Begleiterkrankungen spielen eine wichtige Rolle. Bereits im Alter von 65 Jahren weist jeder 2. Mann mindestens eine chronische Erkrankung auf. Diese Zahl steigt mit zunehmendem Lebensalter weiter an, so dass bei den über 75-jährigen Männern in $>75 \%$ zwei oder mehr Komorbiditäten vorliegen. Der Charlson-Komorbiditätsindex wird vielfach für die Einschätzung der Schwere bestehender Begleiterkrankungen herangezogen und ist ein einfach zu verwendendes Instrument um das allgemeine Sterblichkeitsrisiko effizient abzuschätzen [15] (Tab. 2). 


\section{$>$ Auch die Eigenständigkeit einer Person und ihre kognitiven Fähigkeiten spielt eine wichtige Rolle}

Neben den rein medizinischen Faktoren spielt aber auch die Eigenständigkeit einer Person und ihre kognitiven Fähigkeiten eine wichtige Rolle und lässt neben der Lebenserwartung eine Abschätzung zu, wie gut ein Patient mit den komplexen Veränderungen der Lebensumstände nach Anlage einer Harnableitung umzugehen lernt. Existierende Fragebögen sind hier hilfreich, um die Hilfsbedürftigkeit bei allgemeinen täglichen Aktivitäten (Körperpflege, Ankleiden, Mobilität, Nahrungsaufnahme u. a.) und die geistige Leistungsfähigkeit bei alltäglichen Verrichtungen (Umgang mit Finanzen, Medikation, Nutzung öffentlicher Verkehrsmittel und Telekomunikationsmittel etc.) einfach zu beurteilen $[16,17]$.

Weniger gut messbar sind demgegenüber die allgemeinen Alterungsveränderungen, die u. a. mit einem verminderten Sphinktertonus mit nachfolgender Inkontinenz einhergehen können.

\section{! Bei geplantem orthotopen Blasenersatz sollte bei älteren Patienten unabhängig vom Erektionsstatus und Geschlecht ein Nervenerhalt angestrebt werden.}

Eine entscheidende Rolle spielt aber auch die Operationstechnik. Gerade bei älteren Patienten besteht vielfach bereits präoperativ eine Erektionsstörung, und viele Operateure verzichten daher auf die aufwendigere nervenerhaltende Operationstechnik. Dennoch konnte eindeutig nachgewiesen werden, dass eine nervenerhaltende Operation durch den besseren Erhalt der funktionellen Harnröhrenlänge und der Harnröhrensensibilität im Bereich der membranösen Harnröhre nicht nur für die Wiedererlangung der Erektionsfähigkeit, sondern auch für die Kontinenzerhaltung und die Fähigkeit die Ersatzblase vollständig entleeren zu können, einen entscheidenden Einfluss hat [14]. Dies gilt sowohl für männliche als auch für weibliche Patienten [18]. Bei geplantem orthotopem Blasenersatz sollte daher immer auch beim älteren Patienten unabhängig von Erektionsstatus und Geschlecht ein Nervenerhalt angestrebt werden.

\footnotetext{
$>$ Die kontinente Harnableitung mit ileozäkalem Pouch bildet eine Alternative zum orthotopen Blasenersatz dar
} 
Kontinente kutane Harnableitungen wie der Ileozäkalpouch mit Nabelstoma stellen eine Alternative dar, wenn die Harnröhre nicht erhalten werden kann und werden von einigen Patienten präferiert, die einen möglichen Urinverlust bei orthotoper Ersatzblase definitiv vermeiden wollen. Vorteil dieser Harnableitungsformen ist die Kontinenzrate von über 93\% [19]. Im Gegenzug müssen alle Patienten sich selbst katheterisieren. Stomastenosen treten bei Appendixstoma in bis zu 23\% der Patienten auf, lassen sich aber einfach durch eine tägliche Selbstbougierung verhindern [20]. Pouchsteine entwickeln 5-20\% der Patienten aufgrund unvollständiger Pouchentleerung, chronischer Infektion, Mukusbildung oder der Verwendung von Staplern bei der Anlage des Kontinenzmechanismus auf. Dennoch bildet die kontinente Harnableitung mit ileozäkalem Pouch eine gute Alternative zum orthotopen Blasenersatz der in geübten Händen zu exzellenten funktionellen Ergebnissen führt.

\section{Schlussfolgerung}

Das altersabhängiges Auftreten von Blasenkarzinomen und die demographische Entwicklung werden in den kommenden Jahren zu einer deutlichen Zunahme an Zystektomien bei älteren Patienten führen. Exzellente onkologische und funktionelle Ergebnisse spielen für den Erhalt der Lebensqualität eine entscheidende Rolle. Damit kommt der Harnableitung eine entscheidende Bedeutung zu. Alter allein stellt keine Kontraindikation für eine kontinente Harnableitung dar. Gute Patientenselektion auf individueller Basis und der Einsatz einer nervenerhaltenden Operationstechnik spielen eine entscheidende Rolle, um exzellente funktionelle Ergebnisse bei älteren Patienten zu erreichen.

\section{Fazit für die Praxis}

- Das Blasenkarzinom gehört zu den 10 häufigsten Krebsarten.

- Die radikale Zystektomie stellt die Therapie der Wahl beim muskelinvasiven Blasenkarzinom dar.

- Der orthotope Blasenersatz stellt bei guter Patientenselektion auch für ältere Patienten eine Option mit exzellenten funktionellen Ergebnissen dar.

- Bei Patienten mit geplant orthotopen Ersatz sollte eine nervenerhaltende Operation angestrebt werden. 
Einhaltung ethischer Richtlinien Interessenkonflikt. M. Spahn und S. Boxler

\section{Literatur}

1. Torre LA, Bray F, Siegel RL et al (2015) Global cancer statistics, 2012. CA Cancer J Clin 65(2):87-108

2. Madersbacher S, Hochreiter W, Burkhard F et al (2003) Radical cystectomy for bladder cancer today - a homogeneous series without neoadjuvant therapy. J Clin Oncol 21(4):690-696

3. Shariat SF, Karakiewicz PI, Palapattu GS et al (2006) Outcomes of radical cystectomy for transitional cell carcinoma of the bladder: a contemporary series from the Bladder Cancer Research Consortium. J Urol 176(6 Pt 1):2414-2422

4. Stein JP, Lieskovsky G, Cote R et al (2001) Radical cystectomy in the treatment of invasive bladder cancer: long-term results in 1,054 patients. J Clin Oncol 19(3):666-675

5. Sieber CC (2007) The elderly patient - who is that? Internist (Berl) 48(11):1190

6. Gschwend JE, Fair WR, Vieweg J (2000) Radical cystectomy for invasive bladder cancer: contemporary results and remaining controversies. Eur Urol 38(2):121-130

7. Froehner M, Brausi MA, Herr HW et al (2009) Complications following radical cystectomy for bladder cancer in the elderly. Eur Urol 56(3):443-454

8. Shabsigh A, Korets R, Vora KC et al (2009) Defining early morbidity of radical cystectomy for patients with bladder cancer using a standardized reporting methodology. Eur Urol 55(1):164-174

9. Wuethrich PY, Vidal A, Burkhard FC (2015) There is a place for radical cystectomy and urinary diversion, including orthotopic bladder substitution, in patients aged 75 and older: results of a retrospective observational analysis from a high-volume center. Urol Oncol pii: S1078-1439(15)00404-4. doi: 10.1016/j

10. Clark PE, Stein JP, Groshen SG et al (2005) Radical cystectomy in the elderly: comparison of clincal outcomes between younger and older patients. Cancer 104(1):36-43

11. Sogni F, Brausi M, Frea B et al (2008) Morbidity and quality of life in elderly patients receiving ileal conduit or orthotopic neobladder after radical cystectomy for invasive bladder cancer. Urology 71(5):919-923 
12. Kassouf W, Hautmann RE, Bochner BH et al (2010) A critical analysis of orthotopic bladder substitutes in adult patients with bladder cancer: is there a perfect solution? Eur Urol 58(3):374-383

13. Saika T, Suyama B, Murata T et al (2001) Orthotopic neobladder reconstruction in elderly bladder cancer patients. Int J Urol 8(10):533-538

14. Kessler TM, Burkhard FC, Perimenis P et al (2004) Attempted nerve sparing surgery and age have a significant effect on urinary continence and erectile function after radical cystoprostatectomy and ileal orthotopic bladder substitution. J Urol 172(4 Pt 1):1323-1327

15. Charlson ME, Pompei P, Ales KL, MacKenzie CR (1987) A new method of classifying prognostic comorbidity in longitudinal studies: development and validation. J Chronic Dis 40(5):373-383

16. Rockwood K, Stadnyk K, MacKnight C et al (1999) A brief clinical instrument to classify frailty in elderly people. Lancet 353(9148):205-206

17. Lawton MP, Brody EM (1969) Assessment of older people: self-maintaining and instrumental activities of daily living. Gerontologist 9(3):179-186

18. Gross T, Meierhans Ruf SD, Meissner C et al (2015) Orthotopic ileal bladder substitution in women: factors influencing urinary incontinence and hypercontinence. Eur Urol 68(4):664-671

19. Wiesner C, Bonfig R, Stein R et al (2006) Continent cutaneous urinary diversion: long-term follow-up of more than 800 patients with ileocecal reservoirs. World J Urol 24(3):315-318

20. Kohl U, Gerharz EW, Weingartner K, Riedmiller H (1996) A simple device in the prevention of recurrent appendico-umbilical stenosis. Br J Urol 77(4):603-604 
Tab. 1 Kontraindikationen für die Anlage einer orthotopen Ersatzblase

Kontraindikation

Tumor der distalen prostatischen Urethra/des Blasenhalses bei der Frau

Eingeschränkte Nierenfunktion (Serumkreatinin $>150 \mu \mathrm{mol} / /$ oder GFR $<50 \mathrm{ml} / \mathrm{min}$ )

Eingeschränkte Leberfunktion

Physische oder psychische Einschränkung zur Durchführung des Selbstkatheterismus

Intestinale Dysfunktionen (Morbus Crohn, Kurzdarmsyndrom)

Stressharninkontinenz, Sphinkterdysfunktion, multiple Harnröhrenstrikturen 
Tab. 2 Der Charlson-Komorbiditätsindex berücksichtigt die Summe relevanter Begleiterkrankung hinsichtlich der Lebenserwartung

\begin{tabular}{|c|c|c|}
\hline Komorbidität & Definition & Bewertung \\
\hline Herzinfarkt & $\begin{array}{l}\text { Hospitalisierung wegen elektrokardiographisch und/oder enzymatisch } \\
\text { nachgewiesenem Herzinfarkt }\end{array}$ & 1 \\
\hline Herzinsuffizienz & $\begin{array}{l}\text { Nächtliche oder durch Anstrengung induzierte Dyspnoe mit Besserung der } \\
\text { Symptomatik unter Therapie }\end{array}$ & 1 \\
\hline $\begin{array}{l}\text { Periphere arterielle Verschlus- } \\
\text { skrankheit }\end{array}$ & $\begin{array}{l}\text { Claudiacatio intermittens, peripherer Bypass, akuter arterieller Verschluss, } \\
\text { abdominaler oder thorakaler Aneurysma }\end{array}$ & 1 \\
\hline Zerebrovaskuläre Erkrankungen & $\begin{array}{l}\text { Transitorische ischämische Attacken oder Apoplex ohne schwerwiegende } \\
\text { Residuen }\end{array}$ & 1 \\
\hline Demenz & Chronisches kognitives Defizit & 1 \\
\hline Chronische Lungenerkrankung & $\begin{array}{l}\text { Pulmonal bedingte Dyspnoe bei leichter oder mäßig schwerer Belastung } \\
\text { ohne Therapie oder anfallsweise Dyspnoe (Asthma) }\end{array}$ & 1 \\
\hline Kollagenose & $\begin{array}{l}\text { Polymyalgia rheumatica, Lupus erythamatodes, schwere rheumatoide Ar- } \\
\text { thritis, Polymyositis }\end{array}$ & 1 \\
\hline Ulkuskrankheit & Patienten, die bereits einmal wegen Ulzera behandelt wurden & 1 \\
\hline Leichte Lebererkrankung & Leberzirrhose ohne portale Hypertonie & 1 \\
\hline $\begin{array}{l}\text { Diabetes mellitus (ohne Endor- } \\
\text { ganschäden) }\end{array}$ & Diabetes mellitus und medikamentöse Therapie & 1 \\
\hline Hemiplegie & & 2 \\
\hline
\end{tabular}




\begin{tabular}{|l|l|l|}
\hline Komorbidität & Definition & Bewertung \\
\hline Mäßig schwere Nierenerkrankung & Dialysepflichtigkeit etc. & 2 \\
\hline Diabetes mellitus (mit Endorgan- & Zurückliegende Hospitalisationen wegen hyperosmolarem Koma oder Ke- & 2 \\
\hline schäden) & toazidose & \\
\hline Tumorerkrankung & Sämtliche solide Tumoren ohne Metastasennachweis innerhalb der letzten & 2 \\
\hline Leukämie & 5 Jahre & \\
\hline Lymphom & Akute und chronische Leukämien & 2 \\
\hline Mäßig schwere Lebererkrankung & Leberzirrhose mit portaler Hypertonie ohne stattgehabte Blutung und Vari- & 3 \\
\hline AlDS & Zenblutung in der Anamnese & 2 \\
\hline Metastasierter solider Tumor & & 6 \\
\hline
\end{tabular}

